\title{
Isolation, cloning and bioinformatics analysis of $\beta$-amyrin 11-oxidase coding sequence from licorice
}

\author{
Zahra Shirazi $^{1}$, Ali Aalami ${ }^{1 *}$, Masoud Tohidfar ${ }^{2}$, Mohammad Mehdi Sohani $^{1}$ \\ ${ }^{1}$ Department of Plant Biotechnology, Faculty of Agricultural Sciences, University of Guilan, Rasht, Iran \\ ${ }^{2}$ Department of Biotechnology, Shahid Beheshti University, Tehran, Iran
}

*Corresponding author: ali_aalami@guilan.ac.ir (Ali Aalami)

\begin{abstract}
Licorice is the roots and stolons of Glycyrrhiza uralensis which have several chemical compounds. Triterpene saponins such as glycyrrhizin and glycyrrhetinic acid and flavonoids like liquiritin, isoliquiritigenin and glabron are main compounds detected in liquorice root. The plant's major constituent is a glycyrrhizin. The $\beta$-amyrin 11 -oxidase catalyzes the sequential two-step oxidation of $\beta$-amyrin in C-11 to produce 11-oxo- $\beta$-amyrin, a possible biosynthetic intermediate between $\beta$-amyrin and glycyrrhizin. In this study, the total RNA was extracted from licorice roots and cDNA synthesis, then PCR products were cloned into pTZ57R/T vector. Sequencing confirmed piece length of $1482 \mathrm{bp}$ that encodes a protein of 493 amino acid residues. The results of alignment showed 99\% similarity to $\beta$ - amyrin sequence of Glycyrrhiza uralensis. Subcellular studies using Softberry and Psort software showed that the activity of this protein is in endoplasmic reticulum. Moreover the protein has a signal peptide and is targeted to the secretory pathway. The results of phylogenetic tree determined most similar amino acid sequence to the CYP88D subfamily of cytochrome $\mathrm{P} 450$. These findings can be used for nucleotide or protein manipulation and transformation.
\end{abstract}

Keywords: CYP88D6, Cytochrome P450, Glycyrrhizin, Phylogeny.

Abbreviations: FDP_farnesyl diphosphate; SQS_squalene synthase; OSCs_oxidosqualene cyclases; bAS_ $\beta$-amyrin synthase; LUS_lupeol synthase; CAS_cycloartenol synthase

\section{Introduction}

Licorice, the roots and stolons of Glycyrrhiza (G. glabra and $G$. uralensis), is widely used in the foods and pharmaceutical industries in various countries (Bi et al., 2010; Tian et al., 2008). A number of components have been isolated from the roots of Glycyrrhiza glabra, including triterpene saponins (glycyrrhizin, soyasaponins), Flavonoids (liquritin, glabrol), isoflavones (glabrene, glabridin), chalcones (isoliquiritin), coumarines (liquocoumarin) and polysaccharides (Wittschier et al., 2009). Licorice contain one of the most important crude drugs in the world called glycyrrhizin, which constitutes up to $14 \%$ of total soluble solids content (Ibanoglu and Ibanoglu, 2000; Nassiri-asl and Hosseinzadeh, 2008; Shibata, 2000). Glycyrrhizin, an oleanane-type triterpene saponin, is about 50 times sweeter than sugar which is being used as a sweetener and flavoring additive in the food industry (Chin et al., 2007). It has a wide range of pharmacological, anti-bacterial and anti-oxidant activities (Varsha et al., 2013), hepato-protective (Chan et al., 2003; Jeong et al., 2002; Kimura et al., 2001), antiulcer (He et al., 2001), antiallergy (Park et al., 2004). anticancer (Fiore et al., 2004; Salvi et al., 2003; Yoon et al., 2005), antiviral activity against various DNA and RNA viruses (Fiore et al., 2008), including HIV (De Clercq, 2000) and severe acute respiratory syndrome-associated coronavirus (Cinatl et al., 2003). Biosynthesis of triterpenoids in licorice starts with conversion of two molecules farnesyl diphosphate (FDP) by the squalene synthase enzyme (SQS) to squalene (Lu et al., 2008). Squalene epoxidase oxidizes the squalene to produce 2,3-oxidosqualene (Seki et al., 2011). In the next stage, oxidosqualene cyclases (OSCs) cyclization of 2,3oxidosqualene, an intermediate of both triterpenes and phytosterols (Haralampidis et al., 2002). In G. glabra, three OSCs: $\beta$-amyrin synthase (bAS), lupeol synthase (LUS) and cycloartenol synthase (CAS) were cloned and characterized. These three enzymes are responsible for the branching biosynthesis step of oleanane-type triterpene saponins (glycyrrhizin and soyasaponins), lupane-type triterpene and phytosterols, respectively (Hayashi et al., 2000; Hayashi et al., 2001). Cytochrome P450s play critical roles in oxidative reactions during the biosynthesis of diverse natural plant products, including terpenoids. $\beta$-amyrin oxidation in $\mathrm{C}-11$ and C-30 positions produced glycyrrhizin, C-22 and C-24 positions led to the soyasaponins production (Hayashi et al., 2000; Hayashi et al., 2001; Hayashi and Sudo, 2009; Seki et al., 2008). $\beta$-amyrin 11-oxidase catalyze two sequential oxidation steps of $\beta$-amyrin at the C-11 position to yield 11oxo- $\beta$-amyrin (Seki et al., 2008). $\beta$-amyrin 11 -oxidase plays a key role in engineering the competitive pathway of glycyrrhizin or soyasaponin production and converts intermediate compound of $\beta$-amyrin to glycyrrhizin. In this study, the complete cDNA of $\beta$-amyrin 11-oxidase was isolated from Glycyrrhiza glabra and then subsequently cloned. The protein sequences compared with other cytochrome P450 family involved in the triterpenoids oxidation. Some features of the protein were predicted using in silico analysis. There is no information about bioinformatics features of $\beta$-amyrin 11-oxidase protein sequence until now. These findings about nucleotide and 
protein of $\beta$-amyrin 11-oxidase can be used for the futhure researches for nucleotide or protein manipulation, metabolite engineering and transformation.

\section{Results}

\section{Molecular cloning of $\beta$-amyrin 11-oxidase from G. glabra}

The PCR was reproduced a 1482 bp fragment using specific primers. Clony PCR test and double digestion with $X b a \mathrm{I}$ and BamHI also showed a $1482 \mathrm{bp}$ band from pTZ57R/T clone. Sequencing results showed that cDNA, encoding $\beta$-amyrin 11-oxidase is completely isolated from Glycyrrhiza glabra. This fragment starts with ATG codon and ends with TAG and encodes a predicted protein with 493 amino acid residues. This sequence was recorded in Genbank (KP851192). The amino acid and nucleotide sequence alignment (BLAST finding) showed that $\beta$-amyrin 11oxidase from G. glabra has 99\% nucleotide (AB433179.1) and 99\% amino acid (B5BSX1.1) similarity compared with G. uralensis. Phylogenetic tree was generated based on a multiple sequence alignment among the deduced protein and other proteins of cytochrome P450 from the CYP88 family. The results showed this protein has the most similarity with cytochrome P450 from the CYP88D subfamily (Fig. 1 and Table 1). Proteins of CYP88 family cytochrome P450 involved in the triterpenoids oxidation.

\section{In silico and physicochemical properties of the $\beta$-amyrin} 11-oxidase protein

The primary characterization analysis of $\beta$-amyrin 11-oxidase protein from G. glabra was performed using ProtParam tool. The molecular formula of the protein was predicted to be $\mathrm{C}_{2540} \mathrm{H}_{3999} \mathrm{~N}_{683} \mathrm{O}_{712} \mathrm{~S}_{24}$ with a relative molecular mass and an isoelectric point of $56266.2 \mathrm{D}$ and 8.51, respectively. Instability index of the protein has been calculated 42 that reveals this protein is an unstable protein (stable proteins have instability parameter $<40$ ) and the protein may need to be processed after translation for more stability. The half-life of the protein in vitro condition was calculated $30 \mathrm{~h}$ because of having methionine at the N-terminal end of it. Aliphatic index, as an important factor in assessing the protein resistant to heat was calculated 94.73. The maximum amount of hydrophobicity was -0.179 and 57 of amino acids had negative charge (Asp $+\mathrm{Glu})$ and 62 of them positive charge (Arg + Lys).

\section{Hydrophobicity properties of the $\beta$-amyrin 11-oxidase protein}

The hydrophobicity pattern was calculated using ProtScale with Kyte and Doolittle method (Kyte and Doolittle, 1982). The lowest score was -3.044 in position 329 (glutamic acid) in polypeptide chain and highest score was 2.611 in position 298 (leucine) that are related with the strongest and weakest locations of hydrophilicity. The hydrophobic and the hydrophilic domain were located above and below the zero line, respectively (Fig. 2).

\section{Secondary structure the $\beta$-amyrin 11-oxidase protein}

Study of the protein secondary structure using SOPMA tool showed that this protein has $231 \alpha$ - helix (46.86\%), 87 extended strand (17.65\%), $33 \beta$-turn (6.69\%), 142 random coil (28.80\%) (Fig. 3).
Subcellular localization of the $\beta$-amyrin 11-oxidase protein

Study of the subcellular localization using Softberry and Psort softwares showed the activity and subcellular localization of this protein is in the endoplasmic reticulum membrane (Fig. 4).

\section{Signal peptide sequence of the $\beta$-amyrin 11-oxidase protein}

The Softberry and TargetP softwares showed the protein has a signal peptide and is located in the secretory pathway (Fig. 4). SignalP-3 also predicted the presence of signal peptide in the protein.

\section{Transmembrane segments of the B-amyrin 11-oxidase protein}

TMHMM tool showed the protein has a transmembrane helix at positions of amino acids 3-21 and amino acids 22-493 are toward outside (Fig. 5).

\section{Active site of the $\beta$-amyrin 11-oxidase protein}

Using Prosite, the active site of $\beta$ - amyrin 11-oxidase protein specified $[\mathrm{FW}]-[\mathrm{SGNH}]-\mathrm{x}-[\mathrm{GD}]-\{\mathrm{F}\}-[\mathrm{RKHPT}]-\{\mathrm{P}\}-\mathrm{C}-$ [LIVMFAP]-[GAD] sequence as a protected pattern of the protein, amino acid cysteine $(\mathrm{C})$ is ligand of iron-hem. This motif included amino acids 432-441 and containing of FGAGSRLCPG (Scan Prosite and Pfam).

\section{Tertiary structure of the $\beta$-amyrin 11-oxidase protein}

Three-dimensional protein structure prediction was performed using I-TASSER software and was analyzed using Chimera software. In structure of this protein, transmembrane helix, hydrophobic amino acids, surface structure and active site were specified (Fig. 6).

\section{Discussion}

$\mathrm{P} 450$ and reductase form the $\mathrm{P} 450$ system are grouped in three main types. The first types are the members of the eukaryotic Cytochrome P450 superfamily, that are related to the membrane of the endoplasmatic reticulum and called "microsomes type" type (Omura, 2010). The results of subcellular localization of the $\beta$-amyrin 11 -oxidase protein showed that this protein belongs to this group. The protein group synthesized by membrane-bound ribosomes and anchored by hydrophobic N-terminus (with around 20 amino acids of the N-terminus) into the membrane (De Montellano, 2005; Lee et al., 2008; Poulos and Meharenna, 2007; Urlacher and Eiben, 2006). The TMHMM tool also showed transmembrane helix at positions of amino acids $3-21$ in $\beta$ amyrin 11-oxidase protein. Different P450 families have low sequence identity $<20 \%$, although their structures have a strong conserved topology and three-dimensional fold. All $\mathrm{P} 450$ s have the same catalytic center, which consists of a heme B molecule, by which the P450s is connected to the protein by a thiolate bond to a highly conserved cysteine residue (Schuckel, 2012). Prosite analysis confirmed that amino acid cysteine (C) is ligand of iron-hem in isolated $\beta$ amyrin 11-oxidase. If this thiolate bond is missing, the active P450s will be shifted to inactive species. Function of CYP88 family proteins is oxidation of triterpenoids (Seki et al., 2008). In this study, the phylogenetic tree was obtained 
Table 1. The detail of protein sequences used in the phylogenetic tree drawing.

\begin{tabular}{lll}
\hline Abb. name & Protein name & Plant species \\
\hline CYP88D6A & Beta-amyrin 11-oxidase & Glycyrrhiza uralensis \\
CYP88D6B & Beta-amyrin 11-oxidase & Glycyrrhiza glabra \\
CYP88A3 & ent-kaurenoic acid hydroxylase & Arabidopsis thaliana \\
CYP88A4 & Ent-kaurenoic acid oxidase 2 & Arabidopsis thaliana \\
CYP88A6 & ent-kaurenoic acid oxidase & Pisum sativum \\
CYP88A7 & ent-kaurenoic acid oxidase & Pisum sativum \\
CYP88A2 & ent-kaurenoic acid oxidase & Cucurbita maxima \\
CYP88D3 & cytochrome P450 88D3 & Medicago truncatula \\
CYP88D5 & cytochrome P450 88D5 & Lotus japonicus \\
CYP88D4 & cytochrome P450 88D4 & Lotus japonicus \\
CYP88D7 & putative cytochrome P450 & Astragalus sinicus \\
CYP88D8 & monooxygenase CYP88D7 & Astragalus sinicus \\
CYP88D2 & putative cytochrome P450 & Medicago truncatula \\
CYP93E3 & monooxygenase CYP88D8 & Glycyrrhiza uralensis \\
\hline
\end{tabular}

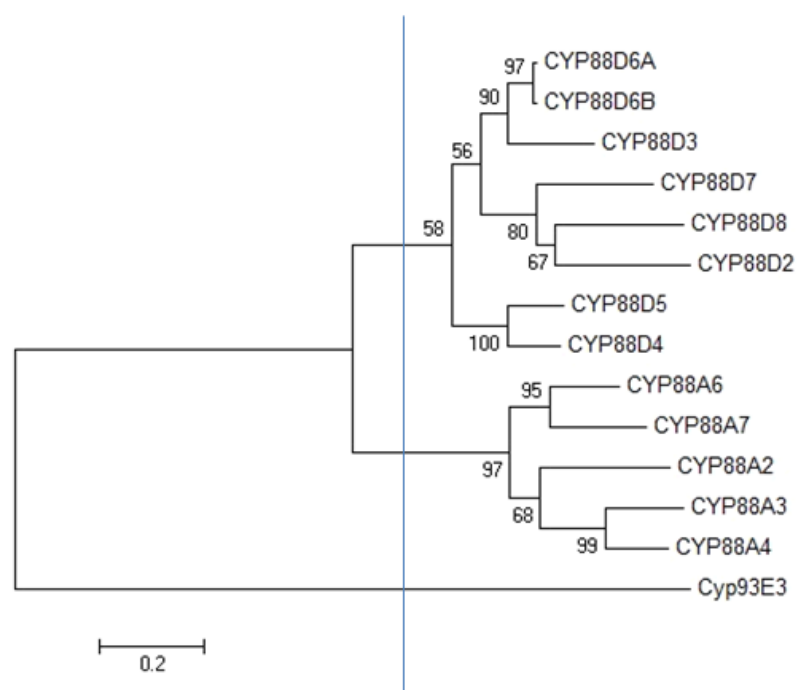

Fig 1. Phylogenetic relationship between amino acid sequence of $\beta$-amyrin 11-oxidase from G. glabra and other CYP88 proteins.

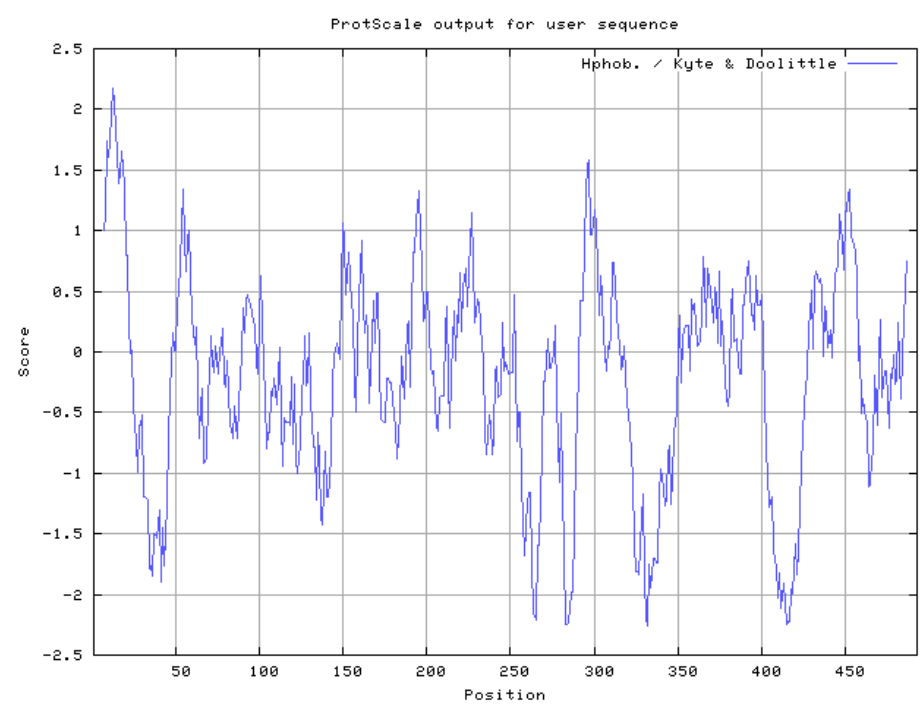

Fig 2. Hydrophobic-hydrophilic pattern of $\beta$-amyrin 11-oxidase protein. Hydrophobic domain is located above the zero line and hydrophilic domain located below the zero line. 

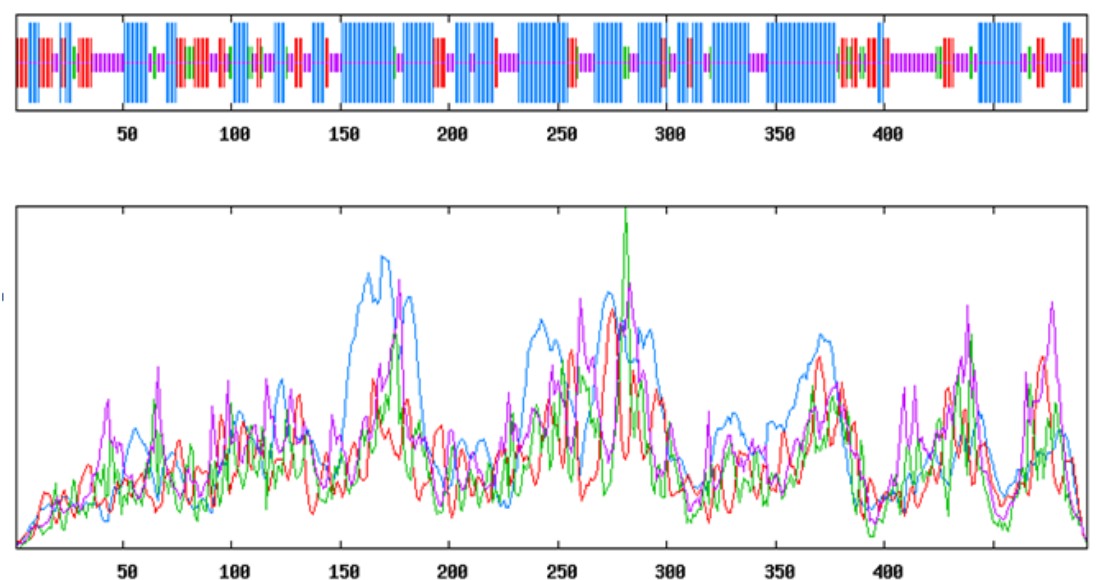

Fig 3. The diagram of secondary structure of $\beta$-amyrin 11-oxidase. $\alpha$-helix (blue lines), Extended strand (red lines), $\beta$-turn (green line) and random coil (purple lines).

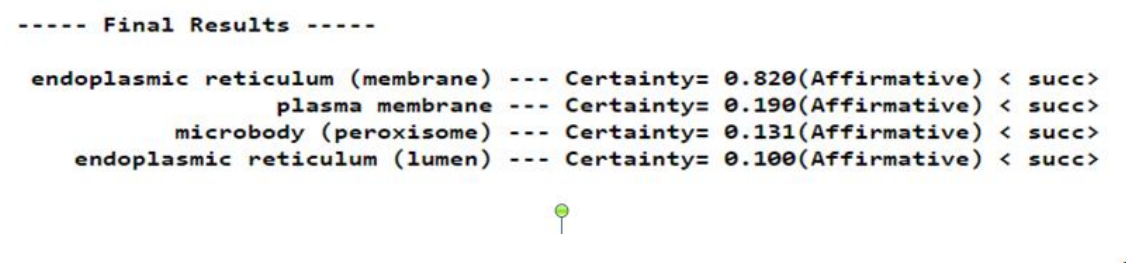

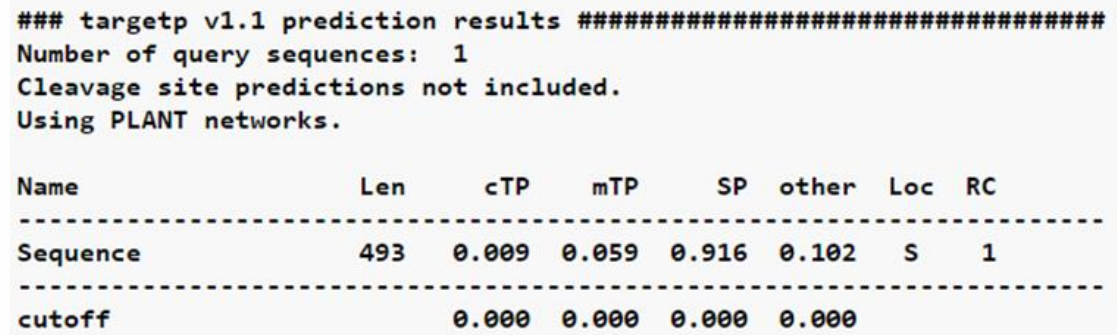

Fig 4. Prediction results of the protein subcellular localization (a) Psort and (b) TargetP , (C: Chloroplast, i.e. the sequence contains cTP, a chloroplast transit peptide; M: Mitochondrion, i.e. the sequence contains mTP, a mitochondrial targeting peptide; S: Secretory pathway, i.e. the sequence contains $\mathrm{SP}$, a signal peptide)

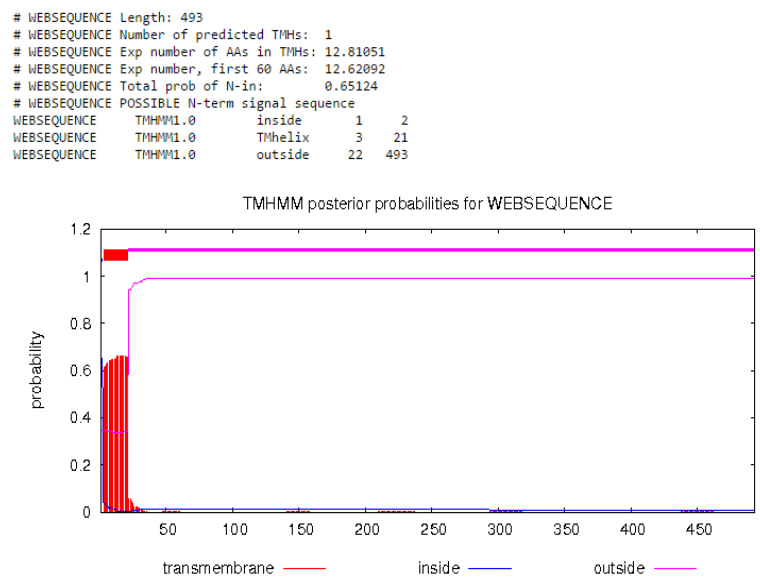

Fig 5. Prediction of transmembrane domain of $\beta$-amyrin 11-oxidase protein using TMHMM. 

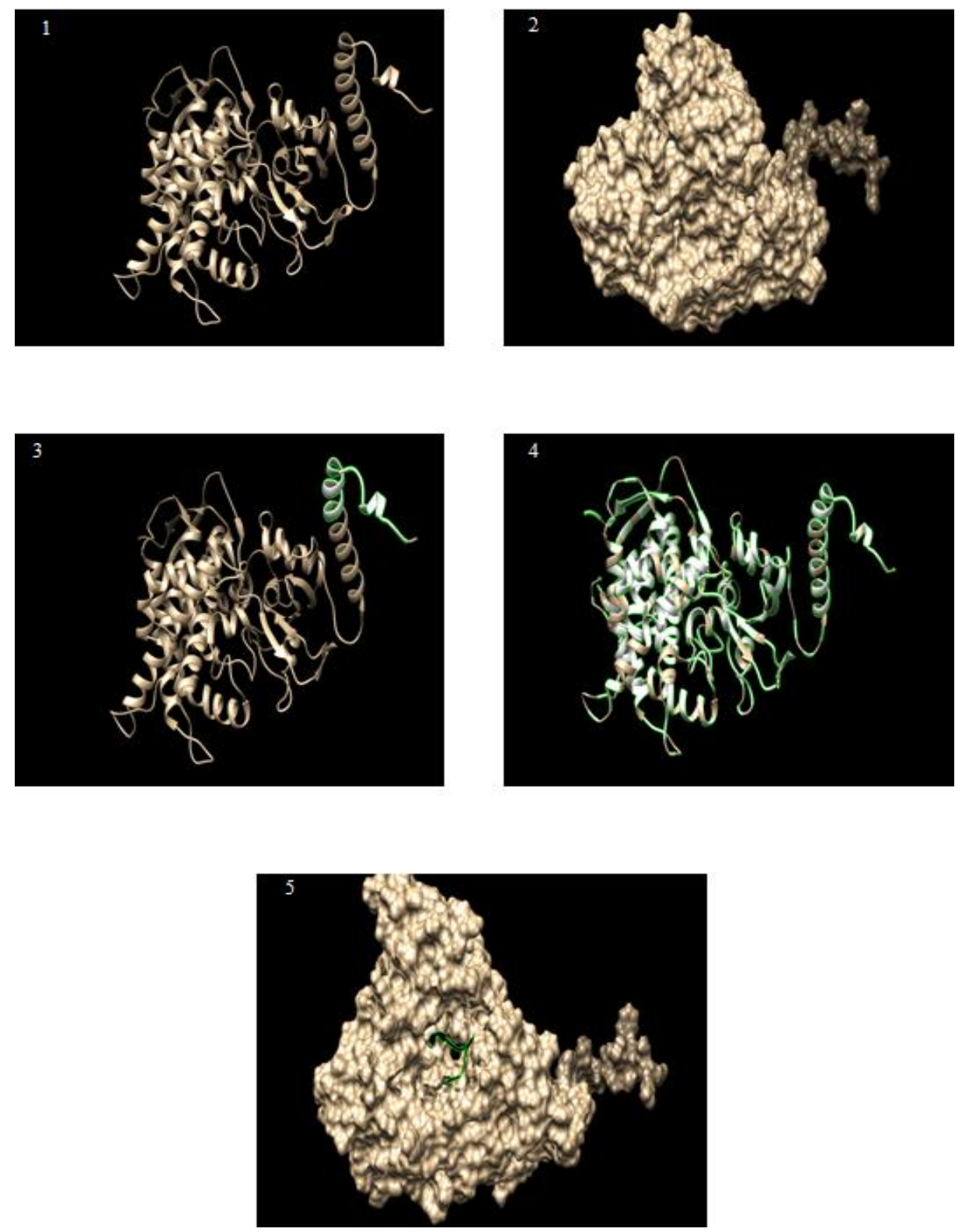

Fig 6. The display of three dimensional structure of $\beta$-amyrin 11-oxidase protein using I-TASSER and its analysis with chimera softwares. Template model (PDB Hit $=4 \mathrm{k} 0 \mathrm{fA}$, Iden $1=0.21$, Iden2=:0.25, Norm. Z-score $=2.90$ ) . .Ident 1 is the percentage sequence identity of the templates in the threading aligned region with the query sequence. Ident 2 is the percentage sequence identity of the whole template chains with query sequence. Cov represents the coverage of the threading alignment and is equal to the number of aligned residues divided by the length of query protein. Norm. Z-score is the normalized Z-score of the threading alignments. Alignment with a Normalized Z-score $>1$ mean a good alignment and vice versa. (1) Three-dimensional structure of the ribbon (2) The surface of protein (3) The transmembrane domain (4) Hydrophobic amino acids (5) The active site of protein

from the CYP88 family and confirmed that CYP88A and CYP88D subfamily were in two separate groups. Some members of the CYP88A subfamily in Arabidopsis, barley and peas are biochemically ent-kaurenoic acid (diterpenoid) oxidase that their functions are in biosynthesis of gibberellin (Davidson et al., 2003; Helliwell et al., 2000). Unlike CYP88A subfamily that is scattered in different plants, it seems that CYP88D subfamily is limited to Fabaceae plants. CYP88D subfamily has activity on $\beta$-amyrin 11 -oxidase or other triterpenoids substrates and each one has a special reaction feature (Seki et al., 2008). This study showed 99\% similarity of this gene between G. uralensis and G. grabla that indicates the same role of this enzyme in the biosynthesis pathway of glycyrrhizin in the two species. The glycyrrhizin is generally found in the wooden parts of thickened roots, while does not exist in the aerial organs, rootlet and root nodules. There is an inverse relationship between glycyrrhizin and soyasaponin (Hayashi, 2009). Soyasaponin observed in all parts of the plant, and in contrast to glycyrrhizin, is mostly observed in younger parts of stolons. 
Both glycyrrhizin and soyasaponins are oleanane-type triterpene that $\beta$-amyrin is the intermediate compound for production both of them (Hayashi et al., 2004). The mRNA of $\beta$-amyrin 11-oxidase from $G$. uralensis is only expressed in the roots and stolons and no expression was observed in the stem and leaves (Seki et al., 2008). Many studies have reported non-production of glycyrrhizin through tissue culture methods (Hayashi, 2009; Hayashi et al., 1998). $\beta$ amyrin oxidation in $\mathrm{C}-11$ and $\mathrm{C}-30$ positions produced glycyrrhizin, which this oxidation is blocked in cell culture and instead $\beta$-amyrin is oxidized in C-22 and C-24 positions and converted to soyasapogenol B. On the other hand, the glycyrrhizin processing is related to the collection of perennial roots from the ground, and in some parts of world, non-harvesting rules have been applied due to indiscriminate harvesting of roots (Yamamoto and Tani, 2005). Therefore, it can be suggested that this gene plays a key role in engineering the competitive pathway of production of glycyrrhizin and soyasaponin and converts intermediate compound of $\beta$-amyrin to glycyrrhizin. Findings of this study resulted better identifying and understanding the characteristics of this enzyme that can help to better engineering of glycyrrhizin biosynthesize pathway.

\section{Materials and Methods}

\section{Plant materials, cDNA synthesis and PCR reaction}

Seeds of Glycyrrhiza glabra were provided by Pakan Bazr Company (Isfahan, Iran). The seeds were soaked in $\mathrm{H}_{2} \mathrm{So}_{4}$ (98\%) for $20 \mathrm{~min}$ and washed with distilled water, then were sown in the small pot. Total RNA was extracted from $G$. glabra fresh root tissue using RNX-plus solution (SinaClonIran). Single-strand cDNA was synthesized using the primers oligo (dT) and reverse transcriptase M-MLV (Thermo Fisher). In order to amplify the encoding region of $\beta$-amyrin 11-oxidase, the forward primer 5'-ATGGAAGTACACTGGGTTTGC-3' and revers primer 5'-CTACGCACATGAAACCTTTATC-3' (SinaClon- Iran) were designed using the Oligo-7 software. Polymerase chain reaction was carried out in total reaction volume of $20 \mu \mathrm{l}$ containing one $\mu 1$ of cDNA, PCR buffer, $3.75 \mathrm{mM}$ of $\mathrm{MgCl} 2,0.5 \mathrm{mM}$ of dNTP, $0.5 \mathrm{pmol}$ of each primers and 0.5 units of Long PCR enzyme (Thermo Fisher). The PCR procedure was as follows: $94{ }^{\circ} \mathrm{C}$ for $5 \mathrm{~min}$; $94{ }^{\circ} \mathrm{C}$ for $1 \mathrm{~min}$, annealing at $55^{\circ} \mathrm{C}$ for $1 \mathrm{~min}$ and extension at $72{ }^{\circ} \mathrm{C}$ for $1: 30 \mathrm{~s}$ ( 35 cycles) and a final extension at $72{ }^{\circ} \mathrm{C}$ for $10 \mathrm{~min}$. After electrophoresis, the amplified fragment from the gel was purified using a DNA extraction kit (Thermo Scientific).

\section{Molecular cloning of $\beta$-amyrin 11-oxidase cDNA}

Ligation was done between the PCR product and pTZ57R/T vector using T4 enzyme (Thermo Fisher). Cloning was performed using E. coli DH5a and The Thermo Fisher InsTAclone PCR Cloning kit. The recombinant clones were selected in LB medium with Ampicillin antibiotic. In order to verify transformed clone, three rapid screening methods (Clony PCR), digestion and sequencing were used. Plasmid was extracted and digested with XbaI and BamHI. Sequencing was performed (Faza pajoh- Iran) using M13 primers in forward and backward directions.

\section{Bioinformatics analysis of the $\beta$-amyrin 11-oxidase}

After sequencing, the chromatogram was studied with chromas ver 2.4 software and DNA Baser ver 4.20 (http://dna-baser-sequence-assembler.soft112.com/). After assurance of appropriate quality of sequences, NCBI database was searched to find similar sequences using the BLAST tool. Obtained amino acid sequence and nucleotide sequence were compared with $G$. uralensis sequence. Multiple Alignment of amino acid sequence and drawing the phylogenetic tree of CYP88 family proteins were performed using the (http://www.ebi.ac.uk/Tools/msa/clustalo/) and MEGA5 (http://www.megasoftware.net/). Protein secondary structure was determined by SOPMA tool (https://npsaprabi.ibcp.fr/) and other characteristics of the protein were determined using the following softwares:

Protparam (http://web.expasy.org/protparam/),

PSORT, Protscale (http://web.expasy.org/protscale/),

Softberry (http://www.softberry.com),

Psort (http://psort.hgc.jp),

TargetP (http://www.cbs.dtu.dk/services/TargetP/), SignalP-3 (http://www.cbs.dtu.dk/services/SignalP), Prosite (http://prosite.expasy.org), TMHMM (http://www.cbs.dtu.dk/services/TMHMM), Scan Prosite (http://prosite.expasy.org/scanprosite/), Pfam (http://pfam.xfam.org),

I-TASSER (http://zhanglab.ccmb.med.umich.edu/ITASSER).

\section{Conclusion}

In this study, $\beta$-amyrin 11 -oxidase cDNA was cloned into pTZ57R/T vector. Sequencing confirmed a fragment with the length of $1482 \mathrm{bp}$ that encodes a protein of 493 amino acid residues. Transmembrane study showed that the protein has a transmembrane helix at positions of amino acids 3-21 and amino. The amino acid cysteine (C), ligand of iron-hem, was found in isolated $\beta$-amyrin 11-oxidase. Subcellular studies showed that the activity of this protein is in endoplasmic reticulum. Moreover, the protein has a signal peptide and is targeted to the secretory pathway. The results of phylogenetic tree determined most similar amino acid sequence to the CYP88D subfamily of cytochrome P450. These findings about of $\beta$-amyrin 11-oxidase can be used by other researchers to work on nucleotide or protein manipulation, metabolite engineering and transformation.

\section{Acknowledgments}

The authors want to express our sincere gratitude to the Plant Biotechnology Department of Guilan University, Rasht, Iran.

\section{References}

Asada Y, Li W, Yoshikawa T (1998) Isoprenylated flavonoids from hairy root cultures of Glycyrrhiza glabra. Phytochem. 47(3):389-392.

Bi W, Tian M, Row KH (2010) Solid-phase extraction of liquiritin and glycyrrhizin from licorice using porous alkylpyridinium polymer sorbent. Phytochem Anal. 21(5):496501.

Chan H-t, Chan C, Ho JW (2003) Inhibition of glycyrrhizic acid on aflatoxin B 1-induced cytotoxicity in hepatoma cells. Toxicology. 188(2):211-217.

Chin Y-W, Jung H-A, Liu Y, Su B-N, Castoro JA, Keller WJ, Pereira MA, Kinghorn AD (2007) Anti-oxidant constituents of the roots and stolons of licorice (Glycyrrhiza glabra). J Agric Food Chem. 55(12):46914697 
Cinatl J, Morgenstern B, Bauer G, Chandra P, Rabenau H, Doerr H (2003) Glycyrrhizin, an active component of liquorice roots, and replication of SARS-associated coronavirus. The Lancet. 361(9374): 2045-2046.

Davidson SE, Elliott RC, Helliwell CA, Poole AT, Reid JB (2003) The Pea Gene NA Encodesent-Kaurenoic Acid Oxidase. Plant Physiol. 131(1):335-344.

De Clercq E (2000) Current lead natural products for the chemotherapy of human immunodeficiency virus (HIV) infection. Medicinal Res Rev. 20(5):323-349.

De Montellano PRO (2005) Cytochrome P450: structure, mechanism, and biochemistry. Springer Science \& Business Media.

Fiore C, Eisenhut M, Krausse R, Ragazzi E, Pellati D, Armanini D, Bielenberg J (2008) Antiviral effects of Glycyrrhiza species. Phytotherapy Res. 22(2):141-148.

Fiore C, Salvi M, Palermo M, Sinigaglia G, Armanini D, Toninello A (2004) On the mechanism of mitochondrial permeability transition induction by glycyrrhetinic acid. Biochimica et Biophysica Acta (BBA)-Bioenergetics. 1658(3):195-201.

Haralampidis K, Trojanowska M, Osbourn AE (2002) Biosynthesis of triterpenoid saponins in plants. In: Scheper $\mathrm{T}$ (ed) History and Trends in Bioprocessing and Biotransformation. Springer, New york.

Hayashi H (2009) Molecular biology of secondary metabolism: case study for Glycyrrhiza plants. In: Kirakosyan A and Kaufman PB (ed) Recent Advances in Plant Biotechnology. Springer, New york.

Hayashi H, Hiraoka N, Ikeshiro Y, Kushiro T, Morita M, Shibuya M, Eizuka Y (2000) Molecular cloning and characterization of a cDNA for Glycyrrhiza glabra cycloartenol synthase. Biol Pharmaceu Bull. 23(2):231234.

Hayashi H, Hiraoka N, Ikeshiro Y, Yamamoto H, Yoshikawa $\mathrm{T}$ (1998) Seasonal variation of glycyrrhizin and isoliquiritigenin glycosides in the root of Glycyrrhiza glabra L. Biological Pharmaceu Bulletin. 21(9):987-989.

Hayashi H, Huang P, Kirakosyan A, Inoue K, Hiraoka N, Ikeshiro Y, KUSHIRO T, Shibuya M, Ebizuka Y (2001) Cloning and Characterization of a cDNA Encoding. BETA.-Amyrin Synthase Involved in Glycyrrhizin and Soyasaponin Biosyntheses in Licorice. Biolo Pharmaceu Bull. 24(8):912-916.

Hayashi H, Huang P, Takada S, Obinata M, Inoue K, Shibuya M, Ebizuka Y (2004) Differential expression of three oxidosqualene cyclase mRNAs in Glycyrrhiza glabra. Biol Pharmaceu Bull. 27(7):1086-1092.

Hayashi H, Sudo H (2009) Economic importance of licorice. Plant Biotech. 26(1):101-104.

He J-X, Akao T, Nishino T, Tani T (2001) The influence of commonly prescribed synthetic drugs for peptic ulcer on the pharmacokinetic fate of glycyrrhizin from ShaoyaoGancao-tang. Biolo Pharmaceu Bull. 24(12):1395-1399.

Helliwell CA, Olive MR, Forster LGR, Peacock WJ, Dennis ES (2000) Isolation of an ent-kaurene oxidase cDNA from Cucurbita maxima. Funct Plant Biol. 27(12):1141-1149.

Ibanoglu E, Ibanoglu s (2000) Foaming behaviour of liquorice (Glycyrrhiza glabra) extract. Food Chem. 70(3):333-336.

Jeong HG, You HJ, Park SJ, Moon AR, Chung YC, Kang SK, Chun HK (2002) Hepatoprotective effects of $18 \beta-$ glycyrrhetinic acid on carbon tetrachloride-induced liver injury: inhibition of cytochrome P450 2E1 expression. Pharmacol Res. 46(3):221-227.
Kimura M, Inoue H, Hirabayashi K, Natsume H, Ogihara M (2001) Glycyrrhizin and some analogues induce growth of primary cultured adult rat hepatocytes via epidermal growth factor receptors. Europ J Pharmacol. 431(2):151161.

Lee D-S, Nioche P, Hamberg M, Raman C (2008) Structural insights into the evolutionary paths of oxylipin biosynthetic enzymes. Nature. 455(7211):363-368.

Lu H-Y, Liu J-M, Zhang H-C, Yin T, Gao S-L (2008) Rimediated transformation of Glycyrrhiza uralensis with a squalene synthase gene (GuSQS1) for production of glycyrrhizin. Plant Mole Biol Rep. 26(1):1-11.

Nassiri-asl M, Hosseinzadeh H (2008) Review of pharmacological effects of Glycyrrhiza sp. and its bioactive compounds. Phytotherapy Res. 26(6):709-724.

Omura T (2010) Structural diversity of cytochrome P450 enzyme system. J Biochem. 147(3):297-306.

Park H-Y, Park S-H, Yoon H-K, Han MJ, Kim D-H (2004) Anti-allergic activity of $18 \beta$-glycyrrhetinic acid-3-O- $\beta$-Dglucuronide. Arch Pharm Res. 27(1):57-60.

Poulos TL, Meharenna YT (2007) Structures of P450 proteins and their molecular phylogeny. Met Ions Life Sci. 3:57-96.

Salvi M, Fiore C, Armanini D, Toninello A (2003) Glycyrrhetinic acid-induced permeability transition in rat liver mitochondria. Biochem Pharmacol. 66(12):23752379.

Schuckel J (2012) Development of a new platform technology for plant Cytochrome P450 fusions. PhD thesis, University of York

Seki H, Ohyama K, Sawai S, Mizutani M, Ohnishi T, Sudo H, Akashi T, Aoki T, Saito K, Muranaka T (2008) Licorice $\beta$-amyrin 11-oxidase, a cytochrome P450 with a key role in the biosynthesis of the triterpene sweetener glycyrrhizin. Proc Natl Acad Sci. 105(37):14204-14209.

Seki H, Sawai S, Ohyama K, Mizutani M, Ohnishi T, Sudo H, Fukushima EO, Akashi T, Aoki T, Saito K (2011) Triterpene functional genomics in licorice for identification of CYP72A154 involved in the biosynthesis of glycyrrhizin. The Plant Cell. 23(11):4112-4123.

Shibata S (2000) A drug over the millennia: pharmacognosy, chemistry, and pharmacology of licorice. J Pharmaceut Soci Jap. 120(10):849-862.

Tian M, Yan H, Row KH (2008) Extraction of glycyrrhizic acid and glabridin from licorice. International journal of molecular sciences 9(4):571-577.

Urlacher VB, Eiben S (2006) Cytochrome P450 monooxygenases: perspectives for synthetic application. Trends Biotech. 24(7):324-330.

Varsha S, Agrawal R, Sonam P (2013) Phytochemical screening and determination of anti-bacterial and antioxidant potential of Glycyrrhiza glabra root extracts. J Environ Res Develop. 7(4A):1552.

Wittschier N, Faller G, Hensel A (2009) Aqueous extracts and polysaccharides from liquorice roots (Glycyrrhiza glabra L.) inhibit adhesion of Helicobacter pylori to human gastric mucosa. J Ethnopharmacology. 125(2):218-223.

Yamamoto Y, Tani T (2005) Field study and pharmaceutical evaluation of Glycyrrhiza uralensis roots cultivated in China (Material study). J Tradition Medicines. 22, 86-97.

Yoon G, Jung YD, Cheon SH (2005) Cytotoxic allyl retrochalcone from the roots of Glycyrrhiza inflata. Chem Pharmaceut Bull. 53(6):694-695. 